OCU-PHYS 263

\title{
Exact Solutions of Einstein-Yang-Mills Theory with Higher-Derivative Coupling
}

\author{
Hironobu Kihara* \\ Osaka City University, Advanced Mathematical Institute (OCAMI), \\ 3-3-138 Sugimoto, Sumiyoshi, Osaka 558-8585, Japan \\ Muneto Nittat \\ Department of Physics, Keio University, Hiyoshi, \\ Yokohama, Kanagawa 223-8521, Japan
}

\begin{abstract}
We construct a classical solution of an Einstein-Yang-Mills system with a fourth order term with respect to the field strength of the Yang-Mills field. The solution provides a compactification proposed by Cremmer and Scherk; ten-dimensional space-time with a cosmological constant is compactified to the four-dimensional Minkowski space with a six-dimensional sphere $S^{6}$ on which an instanton solution exists. The radius of the sphere is not a modulus but is determined by the gauge coupling and the four-derivative coupling constants and the Newton's constant. We also construct a solution of ten-dimensional theory without a cosmological constant compactified to $\mathrm{AdS}_{4} \times S^{6}$.
\end{abstract}

*Electronic address: kihara(at)sci.osaka-cu.ac.jp

${ }^{\dagger}$ Electronic address: nitta(at)phys-h.keio.ac.jp 
Unification of fundamental forces with space-time and matter often requires higherdimensional space-time rather than our four-dimensional Universe. The early Kaluza-Klein theory unifies gravity and the electro-magnetic interaction by considering five-dimensional space-time with one direction compactified into a circle $S^{1}[1]$. This old idea has been revisited several times. After supergravity was discovered many people tried to unify all forces and matter in higher-dimensional space-time compactified on various internal manifolds [2]. String theory was proposed as the most attractive candidate of unification, but it is defined only in ten-dimensional space-time. In order to realize four-dimensional Universe one has to find a suitable six-dimensional internal space. So many candidates of such spaces were proposed; Calabi-Yau manifolds and orbifold models. Internal manifolds can be deformed with satisfying the Einstein equation, and these degrees of freedom are called the moduli. The moduli introduce unwanted massless particles in four-dimensional world. Recently a new mechanism has been suggested to fix these moduli by turning on the Ramond-Ramond flux on the internal space [3, 4]. This flux compactification has been extensively studied in these years.

We would like to revise the compactification scenario with fixed moduli proposed by Cremmer and Scherk long time ago [5] (see also [6, 7]) in a theory with a cosmological constant. By placing solitons on a compact internal space they showed decompactifying limit with large radius of the internal space is disfavored and the radius is fixed to a certain value determined by coupling constants. They considered the 't Hooft-Polyakov monopole [8] on $S^{2}$ and the Yang-Mills instanton [9] on $S^{4}$, both of which can satisfy, with proper coupling constants, the first order (self-dual) equations rather than the second order equations of motion, but their solutions on higher dimensional sphere are not the case. Since string theory is defined in ten dimensions, it is natural to consider this scenario with stable BPS solitons on a six-dimensional internal space like $S^{6}$.

Higher dimensional generalization of self-dual equations was suggested by Tchrakian some years ago [10]. Eight dimensional case is known as octonionic instantons [11]. Though several works have been done for generalized self-dual equations [12, 13], a six-dimensional case was not discussed because of the lack of conformal property. Recently we have found a new solution to the generalized self-dual equations in an $\mathrm{SO}(6)$ pure Yang-Mills theory with a fourth order term with respect to the field strength of the Yang-Mills field (a four-derivative term) on a six-dimensional sphere $S^{6}$ [14]. 
In this letter we propose to use this solution in the context of a compactification of the Cremmer-Scherk type. In our model ten-dimensional space-time with (without) a cosmological constant is compactified to a four-dimensional Minkowski space $M_{4}$ (anti de Sitter space $\mathrm{AdS}_{4}$ ) with a six-dimensional sphere $S^{6}$, where dimensionality of the internal space, six, is required by the four derivative term. Unlike the case of the absence of gravity [14] the four-derivative coupling constant $\alpha$ can differ from the constant $\beta$ in the generalized self-dual equations. When the relation $\alpha=\beta$ holds the generalized self-dual equations become the Bogomol'nyi equations and solutions are BPS. We find for both $M_{4} \times S^{6}$ and $\operatorname{AdS}_{4} \times S^{6}$ that certain relations exist between the radius of $S^{6}$, the gauge coupling, the four-derivative coupling $\alpha$ and the gravitational coupling constants. When the four-derivative coupling constant $\alpha$ vanishes in the case of $M_{4} \times S^{6}$, these relations reduce to those of the original work by Cremmer and Scherk. The advantage of our model to the Cremmer-Scherk model is that the Yang-Mills soliton in our model satisfies the self-dual equations (the Bogomol'nyi equations for $\alpha=\beta$ ) rather than usual equations of motion in the case of the Cremmer-Scherk model. This ensures the stability of configuration at least for the sector of Yang-Mills fields.

Let us consider that space-time is a ten-dimensional manifold. We consider an EinsteinYang-Mills theory. Our action contains as dynamical variables the Yang-Mills (gauge) fields $A_{\hat{\mu}}^{[a b]}$ and a graviton field or the metric $\hat{g}_{\hat{\mu} \hat{\nu}}$. Indices with a hat "«" will refer to a tendimensional space-time $\left(X^{0}, X^{1}, \cdots, X^{9}\right)$. Latin indices $(a, b, \cdots)$ run from 1 to 6 and refer to an internal space. The Clifford algebra associated with the orthogonal group $\mathrm{SO}(6)$ is useful and we represent generators of the Lie algebra so(6) as their elements. The Clifford algebra is defined by gamma matrices $\left\{\Gamma_{a}\right\}$ which satisfy the following anti-commutation relations, $\left\{\Gamma_{a}, \Gamma_{b}\right\}=2 \delta_{a b}$. These matrices can be realized as $8 \times 8$ matrices with complex coefficients. The generators of $\mathrm{so}(6)$ are represented by $\Gamma_{a b}=\frac{1}{2}\left[\Gamma_{a}, \Gamma_{b}\right]$. We often abbreviate the Yang-Mills fields as $A_{\hat{\mu}}=\frac{1}{2} A_{\hat{\mu}}^{[a b]} \Gamma_{a b}$ and we also use notations with differential forms. Thus the gauge fields are expressed as $A=A_{\hat{\mu}} d X^{\hat{\mu}}$. In this notation, the corresponding field strength $F$ is written as $F=d A+e A \wedge A$, where $e$ is a gauge coupling. Covariant derivative $\mathcal{D}_{\hat{\mu}}$ on an adjoint representation $Y=\frac{1}{2} Y^{[a b]} \Gamma_{a b}$ is defined as $\mathcal{D}_{\hat{\mu}} Y=\partial_{\hat{\mu}} Y+e\left(A_{\hat{\mu}} Y-Y A_{\hat{\mu}}\right)$, where $Y$ is a scalar multiplet. The action $\mathcal{S}_{\text {total }}$ consists of two parts. One is the EinsteinHilbert action $\mathcal{S}_{E}$ and the other $\mathcal{S}_{Y M T}$ is a Yang-Mills action with a term which is the fourth power of the field strength $F$. Such a quartic term has been studied by Tchrakian [10] and 
so we call it the Tchrakian term. The total action is:

$$
\begin{aligned}
\mathcal{S}_{\text {total }} & =\mathcal{S}_{E}+\mathcal{S}_{Y M T}, \quad \mathcal{S}_{E}=\frac{1}{16 \pi G} \int d v \mathcal{R}, \\
\mathcal{S}_{Y M T} & =\frac{1}{16} \int \operatorname{Tr}\left\{-F \wedge * F+\alpha^{2}(F \wedge F) \wedge *(F \wedge F)-V_{0} d v\right\} .
\end{aligned}
$$

Here the 10 -form $d v$ is an invariant volume form with respect to the metric $\hat{g}$ and is written as $d v=\sqrt{-\hat{g}} d^{10} X$ in a local patch. The scalar curvature is denoted by $\mathcal{R}$. The asterisk "*" denotes the Hodge dual operator. This operator defines an inner product over differential forms, and for a given form $\omega, \omega \wedge * \omega$ is proportional to the invariant volume form $d v{ }^{1}$ The parameters of this action are the Newton's gravitational constant $G$, the gauge coupling $e$, the four-derivative coupling $\alpha$ and the cosmological constant $V_{0}$.

We show the explicit form of the Yang-Mills part with components of $A$ and $F$,

$$
\begin{aligned}
\mathcal{S}_{Y M T}= & -\int d v\left\{\frac{1}{8} F_{\hat{\mu} \hat{\nu}}^{[a b]} F^{\hat{\mu} \hat{\nu},[a b]}+\frac{\alpha^{2}}{8} \tilde{T}_{\hat{\mu} \hat{\nu} \hat{\rho} \hat{\sigma}}^{[a b c d]} T^{\hat{\mu} \hat{\nu} \hat{\rho} \hat{\sigma},[a b][c d]}+\frac{\alpha^{2}}{3 \cdot 16} S_{\hat{\mu} \hat{\nu} \hat{\rho} \hat{\sigma}} S^{\hat{\mu} \hat{\nu} \hat{\rho} \hat{\sigma}}+\frac{1}{2} V_{0}\right\}, \\
F= & \frac{1}{4} F_{\hat{\mu} \hat{\nu}}^{[a b]} d X^{\hat{\mu}} \wedge d X^{\hat{\nu}} \Gamma_{a b}, \quad S_{\hat{\mu} \hat{\nu} \hat{\rho} \hat{\sigma}}=F_{\hat{\mu} \hat{\nu}}^{[a b]} F_{\hat{\rho} \hat{\sigma}}^{[a b]}+F_{\hat{\mu} \hat{\rho}}^{[a b]} F_{\hat{\sigma} \hat{\nu}}^{[a b]}+F_{\hat{\mu} \hat{\sigma}}^{[a b]} F_{\hat{\nu} \hat{\rho}}^{[a b]}, \\
& T_{\hat{\mu} \hat{\nu} \hat{\rho} \hat{\sigma}}^{[a b][d]}=\left(F_{\hat{\mu} \hat{\nu}}^{[a b]} F_{\hat{\rho} \hat{\sigma}}^{[c d]}+F_{\hat{\mu} \hat{\rho}}^{[a b]} F_{\hat{\sigma} \hat{\nu}}^{[c d]}+F_{\hat{\mu} \hat{\sigma}}^{[a b]} F_{\hat{\nu} \hat{\rho}}^{[c d]}\right), \\
& \tilde{T}_{\hat{\mu} \hat{\nu} \hat{\rho} \hat{\sigma} \hat{\sigma}}^{[a b c d]}=\frac{1}{6}\left(T_{\hat{\mu} \hat{\nu} \hat{\rho} \hat{\sigma}}^{[a b b][c d]}+T_{\hat{\mu} \hat{\nu} \hat{\rho} \hat{\sigma}}^{[a c][d b]}+T_{\hat{\mu} \hat{\nu} \hat{\rho} \hat{\sigma}}^{[a d][b c]}+T_{\hat{\mu} \hat{\nu} \hat{\rho} \hat{\sigma}}^{[c d][a b]}+T_{\hat{\mu} \hat{\nu} \hat{\rho} \hat{\sigma}}^{[d b][a c]}+T_{\hat{\mu} \hat{\nu} \hat{\rho} \hat{\sigma}}^{[b c][a d]}\right) .
\end{aligned}
$$

The Euler-Lagrange equations from these actions read the usual Einstein equation and the equations for the Yang-Mills fields:

$$
\mathcal{R}_{\hat{\mu} \hat{\nu}}-\frac{1}{2} \hat{g}_{\hat{\mu} \hat{\nu}} \mathcal{R}=8 \pi G \mathcal{T}_{\hat{\mu} \hat{\nu}}, \quad \mathcal{D}_{\hat{\mu}}\left[\sqrt{-g} F^{\hat{\mu} \hat{\nu}}-2 \alpha^{2} \sqrt{-g} F^{[\hat{\mu} \hat{\nu}} F^{\hat{\rho} \hat{\sigma}]} F_{\hat{\rho} \hat{\sigma}}\right]=0
$$

Here the energy-momentum tensor $\mathcal{T}_{\hat{\mu} \hat{\nu}}$ is obtained by the variation of the Yang-Mills part with respect to the metric:

$$
\begin{aligned}
\mathcal{T}_{\hat{\mu} \hat{\nu}} & =\frac{1}{2} F_{\hat{\mu}}^{\hat{\rho},[a b]} F_{\hat{\nu} \hat{\rho}}^{[a b]}+\alpha^{2} \tilde{T}_{\hat{\mu} \hat{\rho} \hat{\sigma} \hat{\tau}}^{[a b c d]} T_{\hat{\nu}}^{\hat{\rho} \sigma \hat{\sigma} \hat{\tau},[a b][c d]}+\frac{\alpha^{2}}{3 \cdot 2} S_{\hat{\mu} \hat{\rho} \hat{\sigma} \hat{\tau}} S_{\hat{\nu}}^{\hat{\rho} \hat{\sigma} \hat{\tau}}-\frac{1}{2} g_{\hat{\mu} \hat{\nu}} \chi \\
\chi & =\frac{1}{4} F_{\hat{\mu} \hat{\nu}}^{[a b]} F^{\hat{\mu} \hat{\nu},[a b]}+\frac{\alpha^{2}}{4} \tilde{T}_{\hat{\mu} \hat{\nu} \hat{\rho} \hat{\sigma}}^{[a b c d]} T^{\hat{\mu} \hat{\nu} \hat{\rho} \hat{\sigma},[a b][c d]}+\frac{\alpha^{2}}{3 \cdot 8} S_{\hat{\mu} \hat{\nu} \hat{\rho} \hat{\sigma}} S^{\hat{\mu} \hat{\nu} \hat{\rho} \hat{\sigma}}+V_{0}
\end{aligned}
$$

To solve these equations, we make an ansatz which is the same as that of Cremmer-Scherk. Our ansatz for the metric is the following:

$$
d s^{2}=\eta_{\mu \nu} d x^{\mu} d x^{\nu}+\frac{\delta_{I J}}{\left(1+y^{2} / 4 R_{0}^{2}\right)^{2}} d y^{I} d y^{J}=\hat{g}_{\hat{\mu} \hat{\nu}} d X^{\hat{\mu}} d X^{\hat{\nu}} \quad, \quad y^{2}=\sum_{a=1}^{6}\left(y^{I}\right)^{2}
$$

\footnotetext{
${ }^{1}$ The Hodge dual operator acting on a differential form on a space with Minkowski signature satisfies the following relation: $\left(F_{\mu \nu} d x^{\mu \nu}\right) \wedge *\left(F_{\rho \sigma} d x^{\rho \sigma}\right)=-F_{\mu \nu} F^{\mu \nu} d v$.
} 
where the coordinates $X$ are the total space-time coordinates. The metric $\eta_{\mu \nu}=\operatorname{diag}(-+$ $++)$ is the Lorentz metric on the four-dimensional Minkowski space. Greek indices without a hat " ", for instance $\mu$ will refer to the first four variables. Capital indices $(I, J, \cdots)$ run from one to six and refer to the compact space. The six-dimensional space is taken as a sphere with a radius $R_{0}$. The Riemann tensor, Ricci tensor and scalar curvature are

$$
R_{J K L}^{I}=\frac{1}{R_{0}^{2}}\left(\delta_{K}^{I} g_{J L}-\delta_{L}^{I} g_{J K}\right), \quad \mathcal{R}_{I J}=\frac{5}{R_{0}^{2}} g_{I J} \quad, \quad \mathcal{R}=\frac{30}{R_{0}^{2}}
$$

The rest components of the curvature tensor vanish. In this space, the Einstein equations in (5) reduce to simple equations,

$$
-\frac{1}{2} \eta_{\mu \nu} \frac{30}{R_{0}^{2}}=8 \pi G \mathcal{T}_{\mu \nu}, \quad 0=\mathcal{T}_{\mu I}, \quad-\frac{1}{2} \frac{20}{R_{0}^{2}} g_{I J}=8 \pi G \mathcal{T}_{I J} .
$$

We now make ansatzes for the gauge fields. We assume that the fields $A$ do not depend on the four-dimensional directions, $\partial_{\mu} A=0$, and they have no four-dimensional components $A_{\mu}=0$. This implies that the field strengths are two forms on the six-dimensional sphere: $A=A_{I}(y) d y^{I}, F=\frac{1}{2} F_{I J} d y^{I} \wedge d y^{J}$. With these ansatzes, the four-dimensional part of the energy-momentum tensor becomes $-\frac{1}{2} \eta_{\mu \nu} \chi$, and the equation reduces to $30 / R_{0}^{2}=8 \pi G \chi$. This equation requires that the $\chi$ is a constant. Suppose that the field strength fulfils the generalized self-dual condition

$$
F=i \beta \gamma_{7} *_{6}(F \wedge F)
$$

where $\beta$ is a real parameter. Here " $*_{6}$ " means the Hodge dual on the six-dimensional sphere. Then the second part of the equations of motion (5) is fulfilled automatically by the relation $\mathcal{D} F=0$, where the exterior covariant derivative is defined as $\mathcal{D} F=d F+e(A \wedge F-F \wedge A)$. In fact we have an explicit solution to the self-dual equation:

$$
A=\frac{1}{4 e R_{0}^{2}} y^{a} e^{b} \Gamma_{a b}, \quad F=\frac{1}{4 e R_{0}^{2}} e^{a} \wedge e^{b} \Gamma_{a b}, \quad \beta=\frac{e R_{0}^{2}}{3} .
$$

Here we identify the internal space index and the sphere index. The energy-momentum tensor of this configuration becomes

$$
\zeta \equiv \frac{\alpha^{2}}{\beta^{2}}, \quad \chi=(1+\zeta) \frac{15}{4 e^{2} R_{0}^{4}}+V_{0}, \quad \mathcal{T}_{I J}=-\frac{1}{2}\left\{(1-\zeta) \frac{5}{4 e^{2} R_{0}^{4}}+V_{0}\right\} g_{I J} .
$$

With these ansatzes we obtain algebraic equations from the Einstein equations:

$$
\frac{30}{R_{0}^{2}}=8 \pi G\left(\frac{1}{2}(1+\zeta) \frac{15}{2 e^{2} R_{0}^{4}}+V_{0}\right), \quad \frac{10}{R_{0}^{2}}=8 \pi G\left((1-\zeta) \frac{5}{8 e^{2} R_{0}^{4}}+\frac{V_{0}}{2}\right),
$$


From these we finally obtain

$$
\frac{1}{\pi G}=\frac{1}{e^{2} R_{0}^{2}}(2+4 \zeta) \quad, \quad V_{0}=\frac{15}{4 e^{2} R_{0}^{4}}(1+3 \zeta) .
$$

When the four-derivative coupling vanishes, $\alpha=0$ and therefore $\zeta=0$, these relations reduce to those of the Cremmer and Scherk [5]. ${ }^{2}$ When the relation $\alpha=\beta$ holds $(\zeta=1)$ our solution saturates the Bogomol'nyi bound and becomes a BPS state. The energy density is given by an integral over $S^{6}$ as follows:

$$
\begin{aligned}
E_{Y M T}^{S^{6}} & =\frac{1}{16} \int_{S^{6}} \operatorname{Tr}\left\{-F \wedge *_{6} F+\alpha^{2}(F \wedge F) \wedge *_{6}(F \wedge F)\right\} \\
& =\frac{1}{16} \int_{S^{6}} \operatorname{Tr}\left(i F \mp \alpha \gamma_{7} *_{6}(F \wedge F)\right) \wedge *_{6}\left(i F \mp \alpha \gamma_{7} *_{6}(F \wedge F)\right) \pm \frac{i}{8} \alpha \int_{S^{6}} \operatorname{Tr} \gamma_{7} F \wedge F \wedge F \\
& \geq \pm \frac{i}{8} \alpha \int_{S^{6}} \operatorname{Tr} \gamma_{7} F \wedge F \wedge F=\mp \frac{1}{2^{3}} \int_{S^{6}} \epsilon_{a b c d e f} F^{[a b]} \wedge F^{[c d]} \wedge F^{[e f]} \equiv \pm \mathcal{Q}
\end{aligned}
$$

where the field strength $F$ has only components along $S^{6}$. If the coupling $\alpha$ is equal to $\beta$, the solution of eq. (10) satisfies the Bogomol'nyi equation and the energy attains the local minimum. We can also consider a system coupled with scalar fields. Suppose that scalar fields $Q^{m}$ transform as a representation of $\mathrm{SO}(6)$. The index $m$ labels the representation space. Let us add an action $\mathcal{S}_{Q}$ of the scalar fields $Q$ with a Higgs potential

$$
\mathcal{S}_{Q}=\frac{1}{2}\left\{\int d v D_{\hat{\mu}} Q^{m} D^{\hat{\mu}} Q^{m}+V\left(Q^{2}\right)\right\} \quad, \quad D_{\hat{\mu}} Q^{m}=\partial_{\hat{\mu}} Q^{m}-\frac{1}{2} i e A_{\hat{\mu}}^{[a b]} R\left(\Gamma_{a b}\right)_{m m^{\prime}} Q^{m^{\prime}}
$$

to $\mathcal{S}_{\text {total }}$. The equations of motion are modified. In general, our solution mentioned above does not satisfy the modified equations any more. However, for the scalars which fulfil the covariantly constant condition $D_{\hat{\mu}} Q^{m}=0$ and attain the absolute minimum $V(Q)=0$, the configurations of $A$ and $g$ in equations (7), (11) are still solutions for the modified equations. Here the constant value of the minimum is shifted to 0 . Thus we can argue the Higgs mechanism around our solutions.

Next we suppose that the four-dimensional part is an anti-de Sitter space $\mathrm{AdS}_{4}$ of radius $R_{A}$. Our ansatz for the metric is the following:

$$
d s^{2}=\eta_{\mu \nu}(x) d x^{\mu} d x^{\nu}+g_{I J}(y) d y^{I} d y^{J}=\hat{g}_{\hat{\mu} \hat{\nu}} d X^{\hat{\mu}} d X^{\hat{\nu}}
$$

\footnotetext{
2 We need to redefine $e$ the half when we compare to the result of $[\underline{5}]$.
} 


$$
\begin{array}{rlrl}
g_{I J}(y) d y^{I} d y^{J}=\frac{\delta_{I J}}{\left(1+y^{2} / 4 R_{0}^{2}\right)^{2}} d y^{I} d y^{J} & y^{2}=\sum_{a=1}^{6}\left(y^{I}\right)^{2}, \\
\eta_{\mu \nu}(x) d x^{\mu} d x^{\nu}=\frac{R_{A}^{2}}{\cos ^{2} \theta}\left(-d \tau^{2}+d \theta^{2}+\sin ^{2} \theta d \Omega^{2}\right) & , & d \Omega^{2}=\frac{|d z|^{2}}{\left(1+|z|^{2} / 4\right)^{2}},
\end{array}
$$

where $z$ parametrizes a whole complex plane. The metric $\eta_{\mu \nu}(x)$ is a maximally symmetric metric on the four-dimensional anti-de Sitter space. The Riemann tensor and the Ricci tensor are

$$
\begin{aligned}
R^{\mu}{ }_{\nu \rho \sigma} & =-\frac{1}{R_{A}^{2}}\left(\delta_{\rho}^{\mu} \eta_{\nu \sigma}-\delta_{\sigma}^{\mu} \eta_{\nu \rho}\right), & \mathcal{R}_{\mu \nu} & =-\frac{3}{R_{A}^{2}} \eta_{\mu \nu} \\
R_{J K L}^{I} & =\frac{1}{R_{0}^{2}}\left(\delta_{K}^{I} g_{J L}-\delta_{L}^{I} g_{J K}\right), & \mathcal{R}_{I J} & =\frac{5}{R_{0}^{2}} g_{I J} .
\end{aligned}
$$

The total scalar curvature is obtained by a summation of those of two parts: $\mathcal{R}=-\frac{12}{R_{A}^{2}}+\frac{30}{R_{0}^{2}}$. In this space, the Einstein equations are

$$
\mathcal{R}_{\mu \nu}-\frac{1}{2} \eta_{\mu \nu} \mathcal{R}=8 \pi G \mathcal{T}_{\mu \nu}, \quad \mathcal{R}_{I J}-\frac{1}{2} g_{I J} \mathcal{R}=8 \pi G \mathcal{T}_{I J}
$$

The ansatz for the gauge fields is the same as previous one and the energy momentum tensor does not change. With these ansatzes, we obtain algebraic equations from the Einstein equations as

$$
\frac{3}{R_{A}^{2}}-\frac{15}{R_{0}^{2}}=-4 \pi G\left\{(1+\zeta) \frac{15}{4 e^{2} R_{0}^{4}}+V_{0}\right\} \quad, \quad \frac{6}{R_{A}^{2}}-\frac{10}{R_{0}^{2}}=-4 \pi G\left\{(1-\zeta) \frac{5}{4 e^{2} R_{0}^{4}}+V_{0}\right\} .
$$

We are interested in a possible relation to string theory and therefore we consider the case with the vanishing cosmological constant, $V_{0}=0$. In this case, the radii $\left(R_{A}, R_{0}\right)$ are written by the couplings,

$$
R_{0}^{2}=(5+7 \zeta) \frac{\pi G}{4 e^{2}}, \quad R_{A}^{2}=\frac{5+7 \zeta}{5+15 \zeta} R_{0}^{2}
$$

Thus the additional higher derivative coupling term of the Tchrakian type does not affect critically to the equations of motion. When $\zeta=1$ our solution becomes a solution of the Bogomol'nyi equation again.

Our solutions introduced in this letter are new solutions of the system with a Tchrakian term. The origin of this term has not been clear so far but it seems rather universal in order to construct solitons with codimensions higher than four: for instance it has played a crucial 
role to construct a finite energy monopole (with codimension five) in a six-dimensional spacetime [13]. Though the parameter $\zeta\left(=\alpha^{2} / \beta^{2}\right)$ is a free parameter, we expect that the system goes to $\zeta=1$ because it becomes BPS. There are several discussions on the (in)stability of

higher-dimensional Yang-Mills theories [15]. To compute the mass spectra of the fluctuations around our solutions is a future work. When the scalar fields $Q^{m}$ are non-trivially coupled, the system may allow BPS composite solitons which are made of solitons with different codimensions, as in the case of usual self-dual Yang-Mills equations coupled to Higgs fields [16].

Finally our solution of $\mathrm{AdS}_{4} \times S^{6}$ may have a relation with D2-branes, and we hope that there exists some impact on AdS/CFT duality [17].

\section{Acknowledgments}

We are grateful to D. H. Tchrakian for various comments. We would like to thank Y. Hosotani, H. Itoyama, Y. Yasui, M. Sakaguchi, T. Oota, T. Kimura, S. Shimasaki and E. Itou. We also thank M. Sheikh-Jabbari for an advice. This work is supported by the 21 COE program "Constitution of wide-angle mathematical basis focused on knots" from Japan Ministry of Education.

[1] G. Nordström, Phys. Z. 15, 504 (1914) arXiv:physics/0702221; T. Kaluza, Sitzungsber. Preuss. Akad. Wiss. Berlin (Math. Phys. ) 1921, 966 (1921); O. Klein, Z. Phys. 37, 895 (1926) [Surveys High Energ. Phys. 5, 241 (1986)].

[2] M. J. Duff, B. E. W. Nilsson and C. N. Pope, Phys. Rept. 130, 1 (1986).

[3] K. Dasgupta, G. Rajesh and S. Sethi, JHEP 9908, 023 (1999) arXiv:hep-th/9908088].

[4] S. Kachru, R. Kallosh, A. Linde and S. P. Trivedi, Phys. Rev. D 68, 046005 (2003) arXiv:hep-th/0301240].

[5] E. Cremmer and J. Scherk, Nucl. Phys. B 108, 409 (1976); Nucl. Phys. B 118, 61 (1977).

[6] Z. Horvath, L. Palla, E. Cremmer and J. Scherk, Nucl. Phys. B 127, 57 (1977).

[7] R. Kerner and D. H. Tchrakian, Phys. Lett. B 215, 87 (1988).

[8] G. 't Hooft, Nucl. Phys. B 79, 276 (1974); A. M. Polyakov, JETP Lett. 20, 194 (1974) [Pisma 
Zh. Eksp. Teor. Fiz. 20, 430 (1974)].

[9] A. A. Belavin, A. M. Polyakov, A. S. Schwarts and Yu. S. Tyupkin, Phys. Lett. B 59, 85 (1975).

[10] D. H. Tchrakian, J. Math. Phys. 21, 166 (1980).

[11] B. Grossman, T. W. Kephart and J. D. Stasheff, Commun. Math. Phys. 96, 431 (1984) [Erratum-ibid. 100, 311 (1985)]; R. V. Buniy and T. W. Kephart, Phys. Lett. B 548 (2002) 97 arXiv:hep-th/0210037.

[12] D. H. Tchrakian, Phys. Lett. B 150, 360 (1985); D. O'Se and D. H. Tchrakian, Lett. Math. Phys. 13, 211 (1987); Z. Ma and D. H. Tchrakian, J. Math. Phys. 31, 1506 (1990).

[13] H. Kihara, Y. Hosotani and M. Nitta, Phys. Rev. D 71, 041701 (2005) arXiv:hep-th/0408068];

E. Radu and D. H. Tchrakian, Phys. Rev. D 71, 125013 (2005) arXiv:hep-th/0502025].

[14] H. Kihara and M. Nitta, arXiv:hep-th/0703166.

[15] S. Randjbar-Daemi, A. Salam and J. A. Strathdee, Phys. Lett. B 124, 345 (1983) [Erratumibid. B 144, 455 (1984)]; O. DeWolfe, D. Z. Freedman, S. S. Gubser, G. T. Horowitz and I. Mitra, Phys. Rev. D 65, 064033 (2002) arXiv:hep-th/0105047; A. E. Mosaffa, S. RandjbarDaemi and M. M. Sheikh-Jabbari, arXiv:hep-th/0612181.

[16] M. Eto, Y. Isozumi, M. Nitta, K. Ohashi and N. Sakai, J. Phys. A 39, R315 (2006) arXiv:hep-th/0602170].

[17] J. M. Maldacena, Adv. Theor. Math. Phys. 2, 231 (1998) [Int. J. Theor. Phys. 38, 1113 (1999)] arXiv:hep-th/9711200; E. Witten, Adv. Theor. Math. Phys. 2, 253 (1998) arXiv:hep-th/9802150]; O. Aharony, S. S. Gubser, J. M. Maldacena, H. Ooguri and Y. Oz, Phys. Rept. 323, 183 (2000) arXiv:hep-th/9905111. 